\title{
A Nonhomogeneous Boundary-Valued Problem for the coupled KDV system
}

\author{
Yitong $\mathrm{Pei}^{1}$ and Boling Guo ${ }^{1}$ \\ ${ }^{1}$ Institute of Applied Physics and Computational Mathematics
}

March 13, 2021

\begin{abstract}
In this paper, we study the initial-boundary-value problem (IBVP) for coupled Korteweg-de Vries equations posed on a finite interval with nonhomogeneous boundary conditions. We overcome the requirement for stronger smooth boundary conditions in the traditional method via the Laplace transform. Our approach uses the strong Kato smoothing property and the contraction mapping principle.
\end{abstract}

\section{Hosted file}

IBVP of coupled kdv.pdf available at https://authorea.com/users/349689/articles/513462-anonhomogeneous-boundary-valued-problem-for-the-coupled-kdv-system

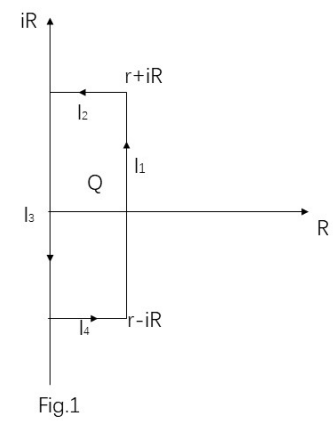

